\title{
Research on the Cognitive Theory and Its Applications on Contemporary Development of English Language and Literature
}

\author{
Peng Sui \\ School Of Foreign Languages, Zhengzhou University Of Industrial Technology, \\ Henan, 451100 China
}

\begin{abstract}
In this paper, we conduct research on cognitive theory and its applications on contemporary development of English language and the literature. The development of cognitive linguistics is not an accident, it reveals a kind of natural law in the development of the literature as is a result of the collision theory of new things, is a kind of literary criticism of the overwhelming trend, however, the creation of a new theory in any must experience a long process of the evolution and development. Cognitive poetics development trend from abroad and also can see emerging discipline localization in the future. Foreign interdisciplinary cognitive poetics is strong, and in the process of its localization, also should apply the principle of psychology, anthropology and other disciplines to the construction of cognitive poetics theory. Under the background, we integrate the related research to propose our new perspective that is meaningful.
\end{abstract}

Keywords-Cognitive Theory, Contemporary, English Language, Literature, Review and Survey.

\section{Introduction}

In recent years, along with the social linguistics have been applied to the language teaching, from the perspective of social linguistics, the language teaching method has also attracted the attention of the linguistic. On the one hand, social linguistics theory has a great revelation on the content and method of foreign language teaching, on the other hand, some of the more modern foreign language teaching method is derived from the social linguistics.

Literary theory does not only exist as abstract theory, but also be applied to the text analysis, for the interpretation of literary works to provide a variety of angles, helps the student to the understanding of the literary history and literary works. Literary theory because of the interdisciplinary, diversity and intelligent characteristics, so it will not only help students understand the history of literature and the literary works, but also help cultivate the students' thinking ability, improve the overall quality of the students. Language cognition study history can be traced back to a long time ago, but the rise of the cognitive linguistics in recent two hundred and thirty years. Language of the cognitive research can be divided into two aspects: one is based on the research of psychology and one is based on the research of linguistics based on the primary research of the psychology focus on language perception, language comprehension, memory, attention, reasoning and some other issues. This research is by means of the laboratory experiments or instrument detection which can think that the language provides intuitive perception of evidence, but they can solve the problem of linguistics is still very limited.

According to the literature survey, the principles of the cognitive theory can be summarized as the following two major aspects. (1) Cognitive linguistics does not accept the distinction between syntax and the semantics of generative grammar. For cognitive linguists, language, the main distinguish not the distinction between syntactic and semantic, but referred to in general language symbols and its communicative functions and can be in form and function, symbol and basic sense of distinction. (2) Function embodied in the structure. A language some structure consisting of a mixture of ingredients symbols, symbols ingredients including word, word markup language, word 
order and intonation language sentence is a basic cognitive schemata and other cognitive schemata belong to the same type of primary and specific cognitive domain [1-3].

Analytical and comprehensive style is two kinds of basic relative language style, but this does not exclude fusion of the two, in a certain sense, analytical style and there is no absolute demarcation line between comprehensive styles. However, comprehensive style, after all, are significant characteristics of literary works, the study of literature language style focuses on comprehensive style is natural. Many language researchers have noticed that the writer's language style and personality have a close relationship and the writer's personality is often expressed by habit of the language expression means. Therefore, to study a writer's language style, the general should begin with analysis of some of his works. In the major works of the author's analysis, it can be found that some of his intercommunity characteristics, so that it can have a base evaluation of his language style. Accordingly, in the figure one, we show the cognitive theory and its primary application scenarios [4].

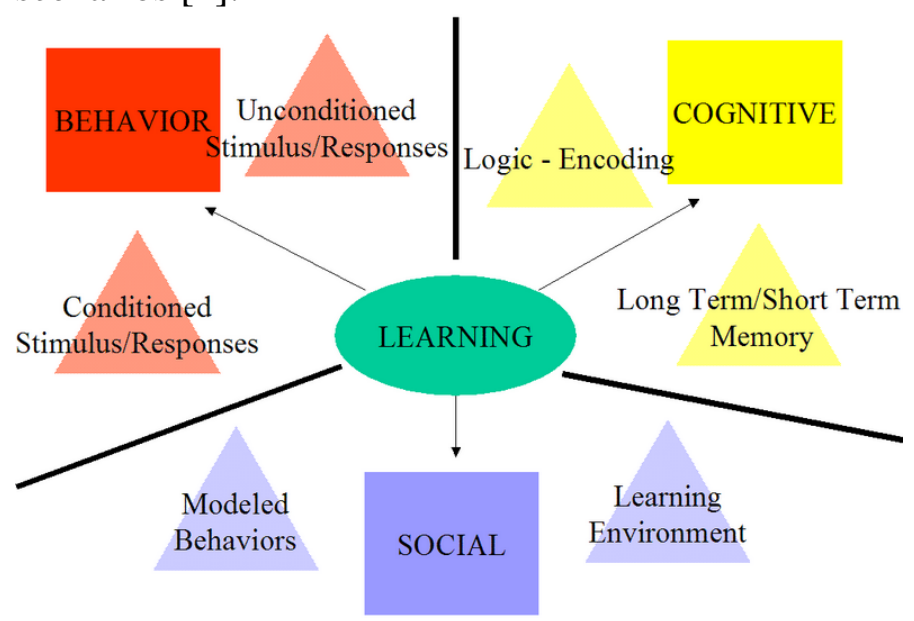

Figure 1. The Cognitive Theory and Its Applications

In this research paper, we analyze the cognitive theory and its applications on the contemporary development of English language and literature. Language variability, discuss and compliance is to ensure that users in the use of language in the process of making choices, from variability and to discuss the selection of the highly flexible, and the result is that the realization of ultimate language compliance throughout the process of basic human complete communicative activities, pragmatics to compliance as a core in the process of language use that will be discussed in the later sections.

\section{The Proposed Methodology}

The Concepts of Cognitive Theory. In two major schools of learning theory of behaviorism and cognitive and behavioral control conditioned stimulus and the link between the observable behavioral responses, pay attention to the nature of the learners' internal psychological structure and cognitive science and change. Deep learning is essentially a structural and the general non-structural knowledge significance construction process is also a complex information processing process, must be obtained for activation of prior knowledge and the depth of the new knowledge effectively and fine processing.

As an important learning theory, cognitive learning theory in several schools are linked to deep learning of the core concepts involved associations, but from the perspective of the core connotation, features and the process of deep learning and constructivism, situated cognition theory, distributed cognition theory and metacognitive theory of the four cognitive theory and deep learning contact more closely. Constructivism from the learning process results and explained the connotation of the deep learning three angles. (1) Cognitive learning theory, the results of the study is to form the cognitive structure as cognitive structure refers to the individual has formed to deal with learning situation or problem situation of inner experience system, can be divided into information systems and operations experience system. As a kind of constructive learning, deep learning obviously is a kind of advanced learning, not only requires 
learners to understand the concept, principle of shallow knowledge, skills, such as structured, more requires learners to understand complex concepts. (2) Deep learning that all knowledge is not exist in isolation, requires the learners to connect new knowledge with the existing knowledge experience, on the basis of the existing knowledge structure to construct new knowledge, as rich or reconstruct the original cognitive structure. (3) As well as the constructive learning and deep learning also occurs in a complex environment, with social environment and learning by creating a meaningful learning environment can help learners to achieve deep and depth of understanding.

In Lewin's psychology theory, there are two basic concepts: mental tension system and living space. Stress refers to a state of system and its surrounding the relationship between the system state, it embodies dynamic characteristics of field; Living space refers to the behavior of the psychological field, people and the environment is regarded as a common power as a whole while highlighting the significance of overall in power. Field theory is that people's behavior is under the influence of people and the environment is the elements of a living space and environment, and only in this environment to human psychological goal with the combination of human environment [5-6].

The English Language and Linguistics. Modern English language expression of the unlimited and the means of communication and communication tools, linguistics research is no longer a pure language basic research, we should pay more attention to research the depth and breadth, which is connected with other subjects, and should be with the interdisciplinary development of the academic research trend. Prototype category theory is that, in the sense of all the relevant language symbols, a more central or core significance, a prototype can be considered as other meaning, the other meaning is on the basis of which further extends or the radiation, thus forming the radiation scope of meaning. Prototype category is a composed of prototype and edge structure. Prototype is typical of the category members and the edge is composed of a member of the central and the category.

Linguistics and philosophy is inseparable, how to see the world depends on how the language. Some scholars said: "linguists work largely on basis of the concept and framework of philosophers, as the starting point". Wittgenstein pay special attention to the words of the speaker and the relationship between the environment to advocate good at from the discourse and environment analysis to identify potential value, and different expressions in different context of discourse, depending on the context determine their significance. In addition, he also attaches great importance to the speaker's language habits, from experience to grasp words and habits of application value and philosophical thinking.

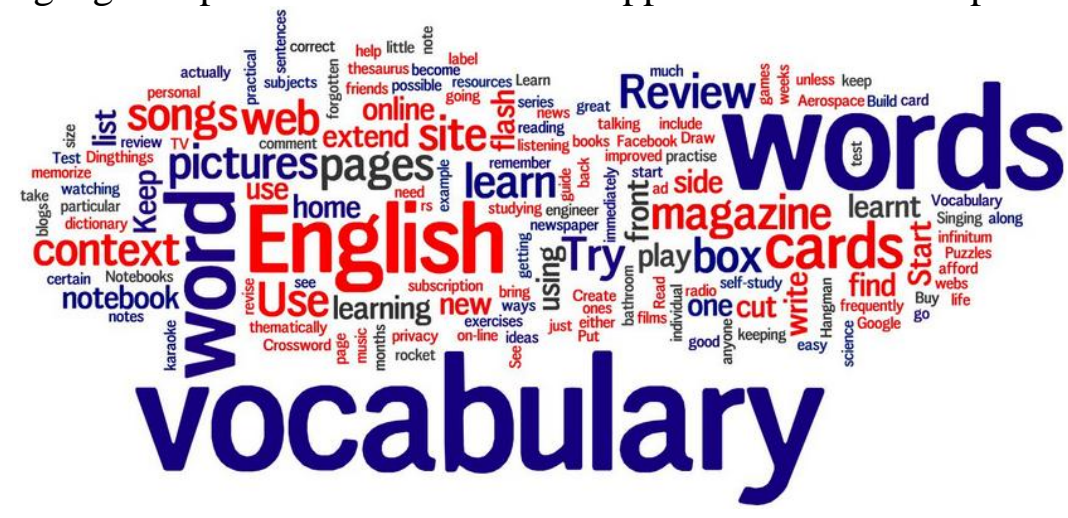

Figure 2. The Keyword of English Language and Linguistics 
Most of the time the language reflects the thoughts and feelings of the speaker, penetrating the speaker's world outlook, the outlook on the life and values from the view of the branch of philosophy, philosophy of English linguistics research perspectives can extend in the field of ethics, aesthetic and general logic. Cognitive linguistics is not simply as a rhetorical metaphor method but see it as a way of thinking to be studied and explained. Conceptual metaphor theory is that metaphor is the mapping of the source domain to target domain which is the system lies in people's concept of system cognitive phenomenon. When a certain kind of language expression in form, length, complexity, as well as the relationship between form elements in parallel to the expression way of coding concept, experience and communication strategy, and we say the language expression has the nature of iconicity.

Practice the theory of cognitive linguistics, therefore, college English vocabulary teaching process is not to explain the cognitive laws, but the process of comprehensive utilization of cognitive law, stress the cognitive process of recreating make students acquire vocabulary. Edge meanings by basic prototype by some form evolved step by step. At every step in the process of evolution, some of the characteristics of lower meanings will retain upper and loss of the other features, the outcome become further away from the prototype. Lower meanings are always to some of the extent, in other words, the meanings on the basis of above and the archetypal meanings is the most basic.

The Language Cognitive. Because the iconicity between language and thinking object, between the signifier and the signified of linguistic sign of natural links, main structure, information structure and cohesion can look after each other, and guarantee the basic advancement of the natural order of discourse semantics, forming pattern of thematic sequencing, different. Language iconicity principle to research the pattern of thematic sequencing, discourse in the semantic development provides a new perspective. In fact, language is not purely on the basis of human is the scholars think that abstract principles can roundly explanation of the independent autonomous systems and the meaning of the language also cannot be limited within the language, and should be mainly comes from the interaction between human and the objective world, that is the result of the user to understand the world. It is an objective reality, physiological basis and body experience, cognitive style, knowledge structure, etc.

Dimension refers to language itself inherent in the three-dimensional structure of each language elements. It not only exists in static language knowledge level, also exists in the level of dynamic use of language and communication. From the macroscopic view, language is made up of sound, form and meaning of the dimension. Language structure in many ways reflects the world people experience in structure, directly reflects the conceptual framework of the people. People tend to group similar conceptual structure expressed in similar syntactic structure with the concept of the different structure expressed in different syntactic structure, the relationship between the language structure and the structure is the syntactic iconicity. The dimension of the language concept is the understanding of the nature of language. The law of language acquisition to the language as the starting point of cognition, on the cognitive operation is given priority to, from the perspective of dimensions and stereo, explore effective cognitive strategies to make the learners in unit time to accumulate knowledge, development capacity and improve the overall intelligence [7-8].

The English Language and Literature Development. English and American literature reflects the national history and culture, the general process of learning English and American literature is to experience another language and the culture of the ecological process of the world, the ecological teaching in British and American literature teaching in terms 
of the original intention of language and literature is apt, because behind communication of language and literature is a collision of different world outlook and values, people, environment and the environment, people and the environment in the eye. Cross-cultural awareness refers to treat with this nationality's cultural differences or conflicts of cultural phenomenon, customs and so on has the correct understanding and the understanding, and cultural phenomenon, customs and habits, such as keep tolerant attitude, on the basis of acceptance and adapt. Cross-cultural awareness is particularly important in the foreign language teaching and the learning is learning a foreign language students to use their learned knowledge when communicating with foreigners should have cultural quality, not only can improve the learning ability that can largely improve the students' interpersonal communication skills and language ability [9].

Due to natural and social elements interaction constructed each person a different perspective and feelings, the thinking is very different with resonance. Particularly noteworthy is that writers multiple angle of view of the characters, each character's clothing accessories, for each character and manners of description and language use are deeply in the branding of the natural and social ecological system.

Under the guidance of the cognitive theory of language migration study highlights the learner's role as a cognitive subject, research restricts the learners' interlanguage system migration occurred or not in the various factors and potential criterion, study how migration as a communicative strategy and the learning strategies used by learners. Therefore, it is connected with form linguistics and formalism is that so-called restricts language service function and related factors of the description and study of the language structure is not important and subordinate.

\section{Conclusion}

In this paper, we conduct research on the cognitive theory and its applications on the contemporary development of the English language and the literature. Cognitive science theory including cognitive linguistics and cognitive psychology, etc., has been applied to the different research areas. When these cognitive science has been applied to the analysis of literary texts, is known as "cognitive poetics" or "cognitive stylistics". Although cognitive poetics theory structure is still in the initial stage, academic theory is a kind of new literary criticism paradigm has practical value. On the premise of theory is not perfect, it still is easy to be used in the analysis of the literature text. On the one hand, scholars can apply early, stoke, and others theory and terminology, on the other hand, we can also be the cognitive sciences, including cognitive linguistics and cognitive psychology theory analysis and research of the literary work. Our research integrates the related theory to enhance the language research.

\section{References}

[1] Tatalovich, Raymond. Nativism reborn?: the official English language movement and the American states. University Press of Kentucky, 2015.

[2] Jimenez-Castellanos, Oscar, and Amelia M. Topper. "The Cost of Providing an Adequate Education to English Language Learners A Review of the Literature." Review of Educational Research 82.2 (2012): 179-232.

[3] Sung, Chit Cheung Matthew. "English as a Lingua Franca and English language teaching: a way forward." ELT journal 67.3 (2013): 350-353.

[4] Hult, Francis M. "English as a transcultural language in Swedish policy and practice." Tesol Quarterly 46.2 (2012): 230-257. 
[5] Erdener, Doğu, and Denis Burnham. "The relationship between auditory-visual speech perception and language-specific speech perception at the onset of reading instruction in English-speaking children." Journal of Experimental Child Psychology 116.2 (2013): 120-138.

[6] Cawthon, Stephanie, et al. "Toward accessible assessments: The promises and limitations of test item adaptations for students with disabilities and English Language Learners." Educational Assessment 18.2 (2013): 73-98.

[7] Gilakjani, Abbas Pourhosein. "The significant role of multimedia in motivating
EFL learners' interest in English language learning." International Journal of Modern Education and Computer Science 4.4 (2012): 57.

[8] Hung, Shao-Ting Alan. "A washback study on e-portfolio assessment in an English as a Foreign Language teacher preparation program." Computer Assisted Language Learning 25.1 (2012): 21-36.

[9] Muftah, Muneera, and Shameem Rafik-Galea. "Error Analysis of Present Simple Tense in the Interlanguage of Adult Arab English Language Learners." English Language Teaching 6.2 (2013): 146. 